\section{Retos para la educación en enfermería}

\author{
Dianne Sofia Gonzalez-Escobar ${ }^{*}$
}

La identificación de las necesidades y los retos a los que debe responder la educación de enfermería deben ir de la mano del conocimiento y análisis de los debates y exigencias a los que se enfrenta la educación superior, como agente de cambio y desarrollo, no solo en los países de América Latina sino a nivel global. Aunque se han logrado enormes avances sobre el conocimiento y la investigación, sectores importantes de la población aún se encuentran sin acceso a los servicios sociales básicos, al empleo, a la salud y a la educación, entre otros. Es por eso, que frente a estas situaciones, la educación superior tiene la responsabilidad de intervenir por ser " un bien público social, un derecho humano y universal, y un deber del Estado; principios que se fundamentan en la convicción profunda de que el acceso, el uso y la democratización del conocimiento es un bien social, colectivo y estratégico, esencial para poder garantizar los derechos humanos básicos e imprescindibles para el buen vivir de nuestros pueblos, la construcción de una ciudadanía plena, la emancipación social y la integración solidaria" (1).

De modo similar, se debe tener en cuenta que a la visión humanista de la educación superior como motor principal del desarrollo humano, que transforme la vida de las personas hacia la paz, la erradicación de la pobreza, el desarrollo sostenible y el diálogo intercultural (1), le corresponde plantearse la necesidad de revisión y transformación de los sistemas educativos, de los procesos de formación y del logro de los aprendizajes. Es por eso, que el mundo actual le exige flexibilidad y creatividad para adaptarse a situaciones cambiantes, teniendo presente que los currículos fijos, inflexibles, rutinarios y estáticos no tienen posibilidad de responder con pertinencia a estos requerimientos (2).

Debe señalarse, que las instituciones de educación superior de los países de América Latina, entre ellas las Colombianas, enfrentan situaciones difíciles en su propósito de responder a su función y responsabilidad social. Es por eso, que les corresponde hacer frente a: las exigencias políticas, demográficas y sociales; a los cambios culturales; a los déficits presupuestales; a las exigencias de modernización e innovación en los procesos pedagógicos y de gestión; a la internacionalización y a la acreditación de calidad, de tal forma que les permita vincularse efectivamente en la sociedad global del conocimiento.

Algunos autores definen los retos de la educación en enfermería desde los procesos que atraviesa la educación superior en general, relacionados con: La masificación de la educación superior, el incremento de la movilidad académica internacional y la migración de profesionales. También depende de la privatización de la educación superior, la crisis de la profesión académica y la educación permanente. Del mismo modo, incide sobre la formación en enfermería, el desarrollo de las tecnologías de la información y la comunicación y su inclusión en la educación (TICs). Igualmente, hay otros factores que la afectan como: la inequidad en el acceso, currículos desactualizados e inflexibles, procesos de evaluación y acreditación; así como la denominada corrupción académica, la competitividad y los ranking, entre otros (3).

La educación en enfermería no es ajena a este contexto y por tanto enfrenta exigencias y retos semejantes relacionados especialmente con la globalización, la responsabilidad de responder a las necesidades sociales y de salud actual; así como el de contribuir al desarrollo de la disciplina de enfermería. Del mismo modo, le corresponde consolidar una práctica profesional reflexiva que permita afrontar las complejas situaciones presentes en los países de América Latina y a nivel mundial; por esta razón, es necesario formar profesionales que piensen local y globalmente y tengan la capacidad de actuar en ambos ámbitos.

Lo anteriormente expuesto, permite reconocer los retos que debe enfrentar la educación de profesionales en enfermería, que se han identificado desde inicios del siglo XXI y están relacionados con: el desarrollo tecnológico, la interconexión, los cambios demográficos y epidemiológicos que evidencian problemas de salud globales. Además, se observan retos como: la persistencia de la inequidad en el acceso y la prestación de servicios de salud en las poblaciones más pobres y vulnerables, la superespecialización de ser-

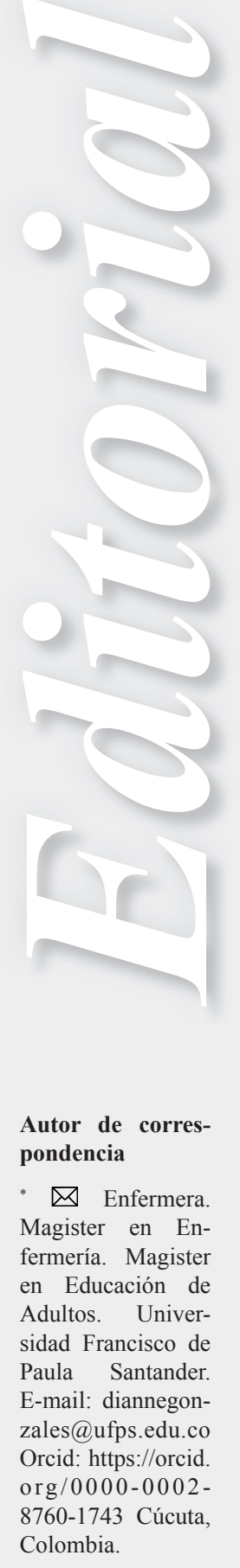


1794-9831

E-ISSN 2322-7028

Vol. 16 No. 2

May - Ago 2019

Cúcuta, Colombia vicios y profesiones. Estas son algunas de las condiciones que sustentan la orientación de las políticas de salud actuales y motivan al logro de los objetivos de desarrollo sostenible. De igual manera, estos desafíos representan el contexto de los problemas y necesidades a los cuales la enfermería debe dar respuesta a través de su práctica profesional, como parte de su compromiso y responsabilidad social, sin alejarse de su esencia.

Por su parte, la Organización Mundial de la Salud (OMS) plantea que el logro de una educación de calidad para los profesionales de la salud del siglo XXI, exige contar con la infraestructura adecuada, con asociaciones y con el diseño de los currículos pertinentes (4); al mismo tiempo, debe estar orientada hacia los principios de la educación transformadora e interprofesional. Entre los principios de la educación transformadora se encuentran la promoción de: el pensamiento crítico y el desarrollo de las aptitudes profesionales necesarias para trabajar en equipo; la adaptación creativa de los recursos mundiales para abordar las prioridades locales; la integración de la educación y los sistemas de salud; la formación de redes y asociaciones; y, el intercambio de recursos educativos y de innovaciones mundiales. Para ello es necesario, que la estrategia para lograr la educación transformadora sea la educación interprofesional, que tiene lugar cuando dos o más profesiones aprenden una acerca de la otra y con cada una, permitiendo la colaboración eficaz y el logro de mejores metas en la atención en salud $(5,6)$. La educación interprofesional promueve el empoderamiento de las enfermeras en el ejercicio de la profesión, aprovechando al máximo su formación y capacitación para trabajar en equipo y utilizar eficientemente los recursos (7).

En la perspectiva que aquí se adopta, es muy significativo tener en cuenta que la globalización y la interdisciplinareidad representan grandes oportunidades para el desarrollo de la disciplina de la enfermería. De igual manera, existen otros factores que son decisivos, tales como: la posibilidad de interactuar con pares de todo el mundo; desarrollar proyectos colaborativos; compartir experiencias; conocer, construir y validar el conocimiento desde visiones diferentes sobre el cuidado del enfermo. Sin embargo, la interdisciplinariedad y el trabajo interprofesional exigen por parte del profesional de la enfermería un sólido y estructurado conocimiento disciplinar, que le permita identificar con claridad su papel dentro del equipo de trabajo para lograr las metas en el cuidado de salud, y así evitar que se pierdan los limites disciplinares diluyendo el conocimiento propio. Desde la formación y la práctica profesional se debe consolidar el conocimiento sobre enfermería, para aplicarlo eficientemente en la atención a los pacientes, a los familiares y al equipo de salud; de igual forma hay que hacerlo efectivo en todos los ámbitos de la práctica profesional y en todos los niveles de atención.

Los planteamientos anteriores permiten prever situaciones a las que la educación en enfermería debe atender desde su naturaleza disciplinar y competencia profesional, no sólo para ser pertinente académica y socialmente, sino porque además tiene la posibilidad de intervenir en muchas de las complejas situaciones que se presentan tanto a nivel local, como regional y mundial. No obstante, es fundamental que estos retos se aborden sin dejar de lado lo que históricamente facilitó el desarrollo y reconocimiento de la enfermería como disciplina y profesión en el área de la salud, siempre en función de:

- Mantener y fortalecer su esencia, continuar construyendo conocimiento que fundamente la práctica profesional, dando legitimidad, autonomía e identidad entre las profesiones del área de la salud.

- Recuperar lo humano del cuidado y la práctica profesional de enfermería, promoviendo los valores que han caracterizado la enfermería en la acción de cuidar: la sensibilidad, solidaridad, respeto por la dignidad, defensa de los derechos humanos, integralidad y responsabilidad.

- Fortalecer la ética profesional en todos los escenarios donde se construye y desarrolla la enfermería como disciplina profesional. Formar sujetos éticos, capaces de ejercer la ciudadanía con responsabilidad, libertad y autonomía, que les permita actuar en el colectivo de forma independiente y participativa, estableciendo juicios de valor y asumiendo con responsabilidad sus elecciones.

Es por tanto conveniente retomar las palabras de Moran para quien "ojalá cualquier institución cuya misión sea la mejora de la formación de los recursos de enfermería, tendamos a lograr las características de una organización de servicio de clase mundial: accesibilidad, competencia, actitud, comunicación, credi- 
bilidad, innovación, capacidad de respuesta, y resultados tangibles, solo de esta manera se podrá cumplir decorosamente con el cometido de coadyuvar a que nuestros estudiantes se conviertan en mujeres y hombres cultivados, que estén a la altura de hacerse cargo de sí mismos y de los demás" (3).

\section{Referencias bibliográficas}

1. III Conferencia Regional Educación Superior (CRES). Declaración y Plan Acción [Internet]. 2018 [Consultado 11 febrero 2019]. Disponible en: www.universidad.edu.co/wp-content/uploads/2018/10/ PropuestaPlanDeAccionCres2018.pdf

2. De Zubiría J. El maestro y los desafíos de la Educación en el siglo XXI. REDIPE VIRTUAL 825. 2013.

3. Moran Peña L. Tendencias en la educación en enfermería: el enfoque desde la internacionalización. Revista Iberoamericana de Educación e Investigación en Enfermería ALADEFE. [Internet]. 2014 [Consultado el 11 de febrero de 2019]; 4(1):5-10. Disponible en: https://www.enfermeria21.com/revistas/aladefe/articulo/101/tendencias-en-la-educacion-en-enfermeria-el-enfoque-de-la-internacionalizacion/

4. World Health Organization. Global Standards for the initial education of professional nurses and midwives [WHO/HRH/HPN/08.6]. [Internet]. Geneva; 2009. [Consultado 18 febrero, 2019]. Disponible en: https://apps.who.int/iris/bitstream/handle/10665/44100/WHO_HRH_HPN_08.6_eng.pdf;jsession $\mathrm{id}=1 \mathrm{C} 379 \mathrm{~B} 7 \mathrm{~F} 13 \mathrm{~A} 78603 \mathrm{C} 764749 \mathrm{~EB} 7087 \mathrm{C} 9 \mathrm{E}$ ? sequence $=1$

5. World Health Organization. Framework for action on interprofessional education \& collaborative practice. [WHO/HRH/HPN/10.3]. [Internet]. Geneva; 2010. [Consultado 14 fDiciembre , 2018]. Disponible en: https://apps.who.int/iris/bitstream/handle/10665/70185/WHO_HRH_HPN_10.3_eng. pdf? sequence $=1$

6. World Health Organization. Interprofessional collaborative practice in primary health care: nursing and midwifery perspectives: six case studies. [Internet]. Human Resources for Health Observer, 13. Geneva; 2013. [Consultado 14 diciembre, 2018]. Disponible en: https://www.who.int/hrh/resources/ IPE_SixCaseStudies.pdf?ua=1

7. Cassiani SHDB, Wilson LL, Mikael SSE, Morán Peña L, Zarate Grajales R, McCreary LL, et al. The situation of nursing education in Latin America and the Caribbean towards universal health. Rev. Latino-Am. Enfermagem. [Internet]. 2017 [Consultado 13 diciembre 2018]; ;25: e2913. Disponible en: http://dx.doi.org/10.1590/1518-8345.2232.2913 